\title{
Tungsten transport and sources control in JET ITER-like wall H-mode plasmas
}

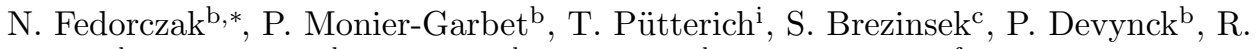

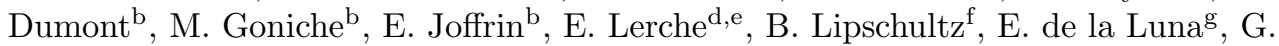 \\ Maddison $^{\text {h}}$, C. Maggi ${ }^{i}$, G. Matthews ${ }^{\mathrm{h}}$, I. Nunes ${ }^{\mathrm{j}}$, F. Rimini ${ }^{\mathrm{h}}$, E.R. Solano ${ }^{\mathrm{g}}$, P. Tamain ${ }^{\mathrm{b}}$, \\ M. Tsalas ${ }^{\mathrm{k}}$, P. de Vries ${ }^{1}$, and JET-EFDA contributors ${ }^{\mathrm{a}}$ \\ ${ }^{a} J E T-E F D A$, Culham Science Centre, Abingdon, OX14 3DB, UK \\ ${ }^{b}$ CEA, IRFM, F-13108 Saint-Paul-Lez-Durance, France \\ ${ }^{c}$ Institute of Energy and Climate Research, Forschungszentrum Jlich, Assoc EURATOM-FZJ, Jlich, \\ Germany \\ ${ }^{d}$ Association EURATOM-Belgian State, LPP-ERM-KMS, TEC partner, Brussels, Belgium \\ ${ }^{e}$ Euratom/CCFE Fusion Association, Culham Science Centre, Abingdon, OX14 3DB, UK \\ ${ }^{f}$ York Plasma Institute, University of York, Heslington, York, YO10 5DD UK \\ ${ }^{g}$ Laboratorio Nacional de Fusin, Asociacin EURATOM/CIEMAT, 28040 Madrid, Spain \\ ${ }^{h}$ Culham Centre for Fusion Energy, EURATOM-CCFE Association, Abingdon, UK \\ ${ }^{i}$ MPI für Plasmaphysik, EURATOM Association, Boltzmannstrasse 2, 85748 Garching, Germany \\ ${ }^{j}$ Istituto de plasmas e fusao nuclear, Lisboa, Portugal \\ ${ }^{k}$ Association EURATOM-Hellenic Republic, NCSR Demokritos 153 10, Attica, Greece \\ ${ }^{l}$ ITER Organization, Route de Vinon sur Verdon, 13115 Saint Paul Lez Durance, France
}

\begin{abstract}
A set of discharges performed with the JET ITER-like wall is investigated with respect to control capabilities on tungsten sources and transport. In attached divertor regimes, increasing fueling by gas puff results in higher divertor recycling ion flux, lower divertor tungsten source, higher ELM frequency and lower core plasma radiation, dominated by tungsten ions. Both pedestal flushing by ELMs and divertor screening (including redeposition) are possibly responsible. For specific scenarios, kicks in plasma vertical position can be employed to increase the ELM frequency, which results in slightly lower core radiation. The application of ion cyclotron radio frequency heating at the very center of the plasma is efficient to increase the core electron temperature gradient and flatten electron density profile, resulting in a significantly lower central tungsten peaking. Beryllium evaporation in the main chamber did not reduce the local divertor tungsten source whereas core radiation was reduced by approximately $50 \%$.
\end{abstract}

PACS: 52.55.Fa, 52.25.Vy, 52.40.Hf

PSI-21: JET, Impurity transport, Boronisation, Fueling, Vertical kicks

*Corresponding author

N. Fedorczak, Cadarache center, Building 508, F-13108 Saint Paul Lez Durance

Email address: nicolas.fedorczak@cea.fr (N. Fedorczak)

Preprint submitted to Elsevier 


\section{Introduction}

Tungsten (W) as divertor material offers both relevant armor life time [1] and minimum fuel retention [2] for a reactor-grade tokamak operation, as tested in JET with the new ITER-like wall (ILW) [3]. The operational drawbacks are possible wall melting [4] under extreme heat load, and pollution of the confined plasma by radiating high-Z ions. As experienced in ASDEX-Upgrade (AUG) $[5 ; 6]$ and now in JET [7], this pollution must be controlled to avoid radiation levels incompatible with high confinement (H-mode) operation, generally at a cost of reduced confinement performances [7]. In AUG, H-modes plasmas are generally performed at non-zero gas puff in the divertor to ensure high edge localized modes (ELMs) frequency, reduce divertor electron temperature $\left(T_{e}\right)$ and thereby the $\mathrm{W}$ source [5]. These ELMs prevent tungsten from entering the core plasma, but also increase the energy and particle losses. Central electron heating is also generally applied to repulse tungsten ions from plasma center. Finally, boronizations are performed to reduce the tungsten source. In this contribution we focus on techniques tested in JET-ILW to control tungsten sources and transport in H-mode attached divertor plasmas. In section 2 , we expose some physics involved in plasma pollution by tungsten ions, linked to the interpretation of experimental data and discharge behavior. In section 3 we present the experimental setup and some aspects of data interpretation concerning tungsten density and source estimate. In section 4 we discuss the experimental observations. First, the influence of divertor gas puff on discharge pollution is investigated in the scope of $\mathrm{W}$ source control, ELMs flushing and divertor screening. The beneficial impact of artificially increased ELMs frequency by magnetic perturbation is discussed. Then, the beneficial impact of main chamber beryllium evaporation is investigated. Finally, core pollution control with central ion cyclotron resonance heating (ICRH) is addressed.

\section{Physics of tungsten source and transport}

Tungsten source originates from physical sputtering of tungsten surfaces by impacting ions. Thermal deuterium ions have a small contribution and light impurities generally dominate: residual carbon, boron and oxygen in the case of AUG [8], beryllium in the case of JET-ILW [9]. Energetic deuterium ions originating from ELMs or neutral beam injection (NBI) can however significantly contribute to tungsten source [10], or ions accelerated in rectified sheath potential in the surrounding of ICRH antennae [11]. Once sputtered, tungsten atoms ionize on a millimeter range by collision with thermal electrons. If ionization occurs within the magnetic pre-sheath of the local surface (case for most erosion areas except maybe for antennae guard limiters), recent experiments [9] and numerical investigations [12] suggest that a large fraction (ordering 100\%) of these tungsten ions are redeposited on the surface without leaving the magnetic pre-sheath. The small fraction (ordering 1\%) of escaping ions spreads along field lines via thermodynamical forces (proportional to parallel temperature gradient) but against friction with main ion parallel flow [13]. Turbulent diffusion [14] transports the ions across flux surfaces up to the separatrix, typically on a millisecond time scale [15]. For source areas located above X-point (main chamber or divertor baffles), transport up to the separatrix is faciliated by the relatively short parallel connection length to the main SOL in contact with the separatrix. On the contrary, divertor sources are better screened due to long 
parallel connection around X-point, accentuated by the divertor collisionality [16].

Once across the separatrix, neoclassical transport is considered to dominate the inward transport of heavy impurity across the pedestal region [14]. Quite generally, the inward pinch increases with density gradient and decreases with temperature gradient. For JET pedestal parameters, the pinch is generally inward through the pedestal and the confined region. Quasi-periodic pedestal relaxation associated to ELMs flush out a significant fraction of ions from the pedestal to the SOL $[17 ; 18]$. By this mechanism, increasing ELM frequency leads to a better pedestal screening of the neoclassical inward pinch $[5 ; 14]$. The relevant parameter there may be linked to the period between ELMs with respect to the pinch time to cross the pedestal width. On the other hand, the excess of particle and energy deposited into the SOL volume by an ELM induces a strong modification of the SOL tungsten flux from the surfaces to the separatrix: (1) the neutral W source is increased $[19 ; 9]$ far above the inter-ELM level; (2) magnetic pre-sheath redeposition may however increase [12]; (3) screening along field line may be reduced [16].

In the core plasma volume inside the pedestal, tungsten ions are strongly ionized and radiate in the XUV range [20]. Photon emissivity is extremely strong in the electron temperature range 1-3 keV, which significantly increases the level of radiated power from inside the separatrix compared to scenarios without tungsten [7]. For a given input power, an increase of core radiation is partly balanced by a decrease of kinetic power losses through separatrix, leading to possible modification of tungsten source and transport in the SOL. It would also tend to decrease the type-I ELM frequency, impacting also the tungsten transport at the edge. Eventually, the level of power losses through separatrix can fall below the high to low (L) confinement mode power threshold and the plasma transits into L-mode. Second, radiation can cause local energy unbalance which results in the local cooling down of thermal electrons. This happens preferentially at the very center of the plasma where tungsten ions tend to cumulate [5; 20]. Once the energy unbalance starts, electron temperature profile flattens, increasing the neoclassical inward pinch, in turns accentuating the particle accumulation at the very center. This vicious cycle leads to an uncontrolled situation eventually ending in a disruption, but which can be counter-acted with sufficient amounts of central electron heating [5]. In ASDEX Upgrade, ECRH and ICRH heating are commonly used for this task [11]. The counterpart of ICRH application is a global increase of core radiation, probably due to an enhanced main chamber tungsten source caused by ICRH antennae polarization. Moreover, MHD activity like saw-teeth also impacts the tungsten transport in core plasmas $[21 ; 22 ; 23]$. A quasi periodic expulsion of particle from the plasma center competes with impurity inward pinch the same manner as ELMs act in the pedestal.

\section{Experimental setup}

\subsection{Plasma scenarios}

Control of tungsten is investigated in two relevant ITER baseline scenarios $\left(q_{95} \approx 3\right)$. The first one relies on ELM frequency control with magnetic perturbations, known as vertical kicks. Under specific conditions, an ELM can be triggered by imposing a fast vertical displacement to the plasma (millisecond time scale). This displacement induces a current perturbation in the pedestal (see [24] for more details), impacting MHD stability. By repeating the perturbation at a given rate, ELM frequency is controlled. This 

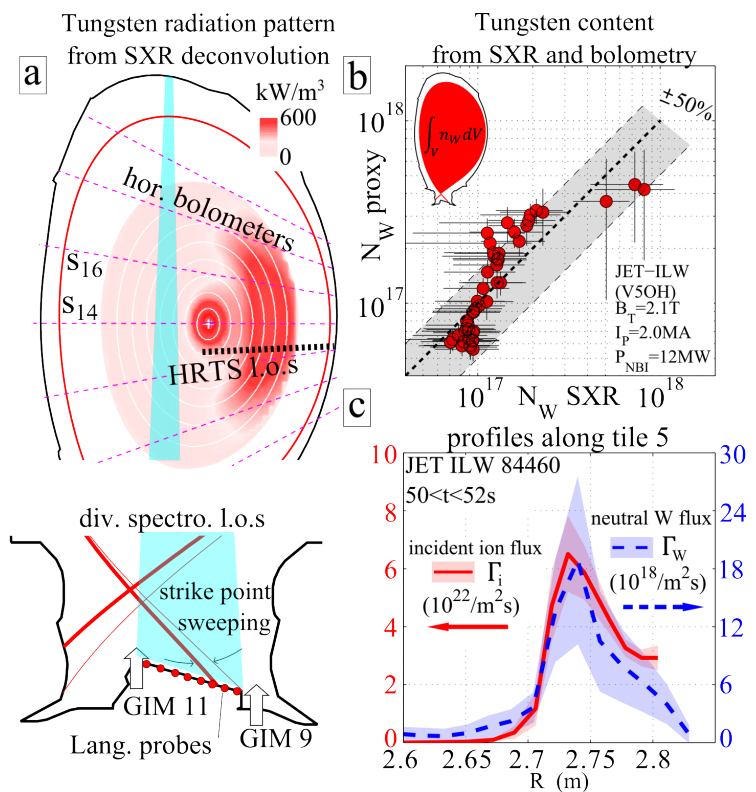

Figure 1: a) JET cross section showing the equilibrium separatrix position, strike points position, and relevant diagnostics geometry. Is also shown a typical tungsten radiation pattern constructed from SXR. b) Tungsten content in the confined volume calculated from SXR compared to its proxy calculated by bolometry, for a series of $10 \mathrm{H}$-mode pulses. Data are time averaged over 1s windows. c) Profiles along tile 5 , of ion flux $\left(\Gamma_{i}\right)$ and tungsten flux $\left(\Gamma_{W}\right.$ using $\left.\frac{S}{X B}=30\right)$ normal to the surface. 
technique is more reliable at low plasma current, which imposes a low magnetic field at fixed safety factor. It is tested on a scenario at $I_{P}=2.0 \mathrm{MA}, B_{T}=2.1 \mathrm{~T}$ on magnetic axis, low plasma triangularity $\delta_{l}=0.36, \delta_{u}=0.19$, pure NBI heating $P_{N B I}=12 \mathrm{MW}$ resulting in a moderate normalized plasma pressure $\beta_{p}=0.5-0.7$. The outer strike point is static on the bulk tungsten tile (tile 5) and the inner strike point on the vertical target (see Fig.1a). Gas fueling is performed in the divertor volume, either in the private flux region (GIM11), or outside the outer strike point (GIM9). ELM frequency is either modified by a shot to shot scan of the fueling rate during the $\mathrm{H}$-mode phase, in the range $\Gamma_{e}=0$ to $10^{22} \mathrm{~s}^{-1}$, or by application of kicks of higher frequency than the natural ELM frequency obtained at a given fueling rate.

A second scenario is considered to investigate the effect of central heating with ion cyclotron frequency waves at $f_{I C F}=42 \mathrm{MHz}$. Plasma current and magnetic field are fixed $I_{P}=2.5 \mathrm{MA}, B_{T}=2.7 \mathrm{~T}$, and the input power is varied both in amplitude and fraction of ICRH $(\max 25 \%) P_{N B I}=12-18 \mathrm{MW}$ and $P_{I C R H}=0-5 \mathrm{MW}$. The minority hydrogen concentration is about $c_{H} \approx 7 \%$, allowing central electron heating for $P_{I C R H} \geq 3 \mathrm{MW}$. Plasma shape is identical as in the previous scenario, except the outer strike point which is radially swept along the bulk tungsten tile (by about $5 \mathrm{~cm}$ at $5 \mathrm{~Hz}$ ) in order to spread the larger heat load. Besides central heating, the gas fueling rate was also scaned to control the ELM frequency and core radiation. In order to optimize the coupling resistance of ICRH antennae, the gas fueling was performed by an arrangement of main chamber nozzles instead of divertor nozzles [25]. This scenario was finally selected to test the consequence of a beryllium evaporation in the main plasma chamber by comparing two series of pulses with identical settings performed before/after the evaporation.

\subsection{Diagnostics}

Tungsten source at the surface of divertor target is monitored by visible spectroscopy [9] with a spatial resolution of about $\Delta R=15 \mathrm{~mm}$. The $W^{0}$ line at $400.9 \mathrm{~nm}$ is fitted out of the background, with a time resolution of about $40 \mathrm{~ms}$. This photon flux can be interpreted in term of particle flux via a collisional-radiative model [26]. The number of ionization per emitted photon, written $\frac{S}{X B}$, is a function of electron temperature [9], so that visible spectroscopy coupled to, for instance, Langmuir probe measurement of $T_{e}$, can yield a quantitative estimate of the flux of tungsten atoms produced at the target. A profile of tungsten influx is shown in Fig.1c, using $\frac{S}{X B}=30$. The tungsten (or photon) flux along the outer divertor tile exhibits a pattern relatively close to the saturation ion flux collected by an array of Langmuir probes (also shown in Fig.1c). To be fully consistent and evaluate the total number of tungsten atoms leaving the surface, the local photon flux should be multiplied by a local $\frac{S}{X B}\left(T_{e}\right)$ value, then integrated over the divertor surface. The time evolution during ELMs should be treated as well. Unfortunately, interpretation of probe measurements with ELM is subject to large errors and generally the time resolution of spectroscopy does not allow the clear distinction of inter-ELM versus intra-ELM tungsten flux. Current investigations suggest that, in the scenarios investigated here (non detached divertor), inter and intra ELMs contributions to the W source are of similar amplitude [27]. For simplicity, signals are averaged over 1 second and we consider a constant and uniform value $\frac{S}{X B}=30$, used across the series of pulses. This yields an effective number of emitted tungsten atoms from the bulk tungsten tile, assumed representative of the tungsten flux emitted by the divertor. Note also that 


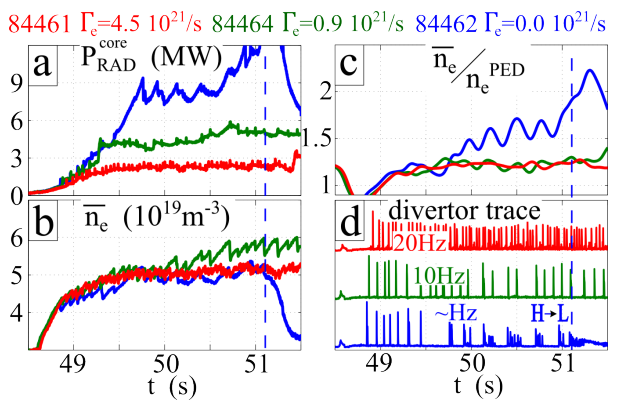

Figure 2: Pulse evolution at different H-mode fueling rates, $B_{T}=2.1 \mathrm{~T}, I_{P}=2.0 \mathrm{MA}, P_{N B I}=12 \mathrm{MW}$. A) radiated power from confined region, b) line averaged electron density, c) density peaking (line averaged density over pedestal density from HRTS), d) ELM traces from divertor

W-components are present in the main chamber of JET-ILW which are not monitored by spectroscopy: the outer and inner divertor baffle made of tungsten coating, and some recessed areas within the beryllium main chamber [28].

The transport of ionized tungsten from the divertor surface up to the separatrix cannot be directly measured in JET. On the other hand, the ionized tungsten density in the confined region can be estimated from soft X-ray (SXR) measurements [29]. After a tomographic reconstruction of the SXR radiation pattern, a bremsstrahlung component is subtracted (see Fig.1a), and deconvoluted into a tungsten density (considered as the only radiator), assuming electron temperature and density uniform on flux surfaces. This tungsten density map is then integrated over the confined plasma volume, with an extrapolation for flux surfaces where $T_{e}<1.5 \mathrm{keV}$, to yield the total number of tungsten atoms in the confined region $N_{W}$. For comparison, we also constructed a proxy for this quantity from the total radiated power $P_{R A D}$ (from the bulk plasma) measured by bolometers, assuming $P_{R A D} \approx N_{W} \bar{n}_{e} L_{W}^{e f f}$ where $\bar{n}_{e}$ is a vertical line averaged electron density measured by interfero-polarimetry and $L_{W}^{e f f}$ is an effective cooling factor for tungsten ions in coronal equilibrium [20]. In the following, we use $L_{W}^{e f f}=5 \cdot 10^{-31} \mathrm{Wm}^{3}$ which stands for the maximum of the cooling factor reached at $T_{e}=1.5 \mathrm{keV}$. As seen in Fig.1c), proxy and SXR estimates of the tungsten amount are in relatively good agreement over one decade, within $50 \%$ error. In the following, the bolometry estimate of the tungsten content is considered, both for its simple expression and its direct proportionality with the core radiation level.

\section{Experimental results}

\subsection{Gas fueling}

Impact of fueling on discharge behavior. Increasing the fueling rate is commonly used in AUG to increase ELM frequency and reduce the W source in order to mitigate the confined plasma radiation caused by tungsten [5]. As shown in Fig.2 and previously reported [7], a finite fueling rate is mandatory in JET-ILW to avoid H-mode collapse by radiation. In the scenario investigated here, a large divertor fueling rate $\left(\Gamma_{e} \approx 15 \times 10^{21} \mathrm{~s}^{-1}\right)$ is applied over typically 1 second prior to H-mode formation, before being reduced to the 
desired value. By this means, early H-mode ELM free phases are avoided. At medium divertor fueling rate $\Gamma_{e}=4.5 \times 10^{21} \mathrm{~s}^{-1}$, ELM frequency is about $f_{E L M}=20 \mathrm{~Hz}$ and the radiated power is stable around $2 \mathrm{MW}$ throughout the $\mathrm{H}$-mode phase. At $\Gamma_{e} \approx 1 \times 10^{21} \mathrm{~s}^{-1}$, ELM frequency falls to about $10 \mathrm{~Hz}$, and the radiated power increases to $5 \mathrm{MW}$ with no sign of collapse after 4s of H-mode. When fueling is switched off during H-mode (except from NBI), ELM frequency drops to a few $\mathrm{Hz}$ and radiated power increases to about 9MW in the first seconds of the $\mathrm{H}$-mode phase. Note that the fast increase of core radiation is associated to an ELM free phase (around $t=49.5 \mathrm{~s}$ ) lasting less than $0.5 \mathrm{~s}$. Concomitant is an increase of the density profile peaking (from pedestal top to core), and a further increase of the radiated power (starting at 50.5s in Fig.2). Although the line averaged density is constant throughout the H-mode plateau, pedestal density continuously falls with low frequency bursts of compounds ELMs, and central density rises, probably due to core cooling. After approximately $2 \mathrm{sec}$ of $\mathrm{H}$-mode, the plasma falls back into L-mode.

Impact of fueling on divertor tungsten influx. The impact of divertor fueling rate on $\mathrm{H}$-mode behavior is summarized in Fig.3 where the trends across series of pulses are illustrated. Instead of fueling rate, the ion recycling flux on the outer divertor target is considered as a marker of divertor regime, (it is collinear with the fueling rate but with different proportionality depending on the injector location). Across the fueling scan, the ion flux varies by a factor of 5 still below detachment, while core electron density from pedestal to core only varies by less than $20 \%$ (except for zero fueling rate as detailed above). As noted previously [7] and shown in Fig.3c, increasing the divertor recycling decreases the tungsten source $\Gamma_{W} \propto \Gamma_{i}^{-0.5(-0.8)}$ for $B_{T}=2.1 \mathrm{~T}\left(B_{T}=2.7 \mathrm{~T}\right)$ scenario. This behavior indicates that the effective sputtering yield decreases by an order of magnitude from $Y_{W} \approx 2 \cdot 10^{-3}$ to $2 \cdot 10^{-4}$ when recycling is increased. The two possible reasons are (1) a change of the impurity content in the divertor, especially of beryllium ions, (2) a change of divertor $T_{e}$ or ion impact energy. Indeed, the power across the separatrix divided by the ion recycling rate, yielding an effective impact energy, falls by a factor of 2.5 across the fueling scan.

Noticeably, the tungsten source is higher and shows more scatter for $B_{T}=2.7 \mathrm{~T}$ than for $B_{T}=2.1 \mathrm{~T}$ conditions. Possible reasons are: (1) difference in the heating scheme: $P_{N B I}=12-18 \mathrm{MW}$ and $P_{I C R H}=0-5 \mathrm{MW}$ for $B_{T}=2.7 \mathrm{~T}$ whereas $P_{N B I}=12 \mathrm{MW}$ and $P_{I C R H}=0$ for $B_{T}=2.1 \mathrm{~T}$, although SOL energy losses are of the same order (Fig.3d). (2) Gaz fueling is from main chamber for $B_{T}=2.7 \mathrm{~T}$ and from divertor only for $B_{T}=2.1 \mathrm{~T}$. (3) The outer strike point is radially swept for $B_{T}=2.7 \mathrm{~T}$ whereas it is static for $B_{T}=2.1 \mathrm{~T}$. (4) ELMs have different energy and particle losses in the two scenarios. Overall, it suggests that a more careful investigation of the $\mathrm{W}$ source mechanisms is needed, especially regarding the variation of divertor conditions and the impact of ELMs.

Impact of fueling on core radiation. As shown in Fig.2b, the total tungsten content (built from the total radiated power from the core) is also decreasing with increased recycling rate $N_{W} \propto \Gamma_{i}^{-1.1(-1.5)}$ for $B_{T}=2.1 \mathrm{~T}\left(B_{T}=2.7 \mathrm{~T}\right)$. It cannot be solely attributed to variation of the source, because their ratio $\tau_{W}=\frac{N_{W}}{\Gamma_{W}}$, either called effective confinement time $[14 ; 30]$ or penetration factor [31], is also decreasing with divertor recycling: $\tau_{W} \propto$ $\Gamma_{i}^{-0.6(-0.7)}$. Assuming that the dominant tungsten source is the outer divertor (which 


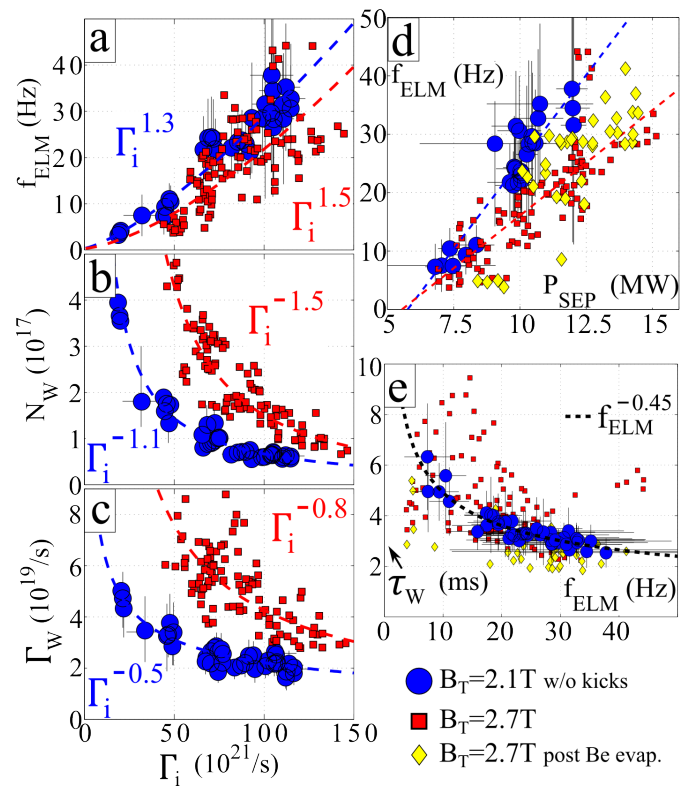

Figure 3: Variation of a) ELM frequency, b) total tungsten content and c) outer divertor tungsten source as a function of outer divertor recycling flux. Dashed curves show the power law trends, with given exponents. d) Variation of ELM frequency vs. power through separatrix, dashed curves are linear trends. e) Variation of tungsten penetration factor vs. ELM frequency. Each point is a 1s average during H-mode. $B_{T}=2.1 \mathrm{~T}$ scenario in blue circles, $B_{T}=2.7 \mathrm{~T}$ scenario before (after) Be evaporation in red squares (yellow diamonds)

may be erroneous, see later), it means that tungsten transport from divertor to the confined region is globally impacted by edge fueling: divertor screening and/or pedestal transport. As stated above, ELM frequency increases with recycling $f_{E L M} \propto \Gamma_{i}^{1.3(1.5)}$ for $B_{T}=2.1 \mathrm{~T}\left(B_{T}=2.7 \mathrm{~T}\right)$. Combining the trends, the tungsten penetration factor decreases with ELM frequency, $\tau_{W} \propto f_{E L M}^{-0.45}$ for $B_{T}=2.1 \mathrm{~T}$ (see Fig.3e), and similarly for $B_{T}=2.7 \mathrm{~T}$ although the data are more scattered in that scenario. This is the same trend has illustrated by Pütterich in [30]

A model of tungsten flushing by ELMs has been proposed by Dux in [14], where an inverse proportionality between $W$ penetration factor and ELM frequency $\tau_{W} \propto f_{E L M}^{-1.1}$ is obtained. The quantitative disagreement with experimental findings may be attributed to the impact of divertor screening, omitted in both modeling and experimental analyses. At this stage, agreement with the model is only qualitative.

Fueling may directly increase the ELM frequency, leading to an increased $\mathrm{W}$ flushing at pedestal. On the other hand, we see from Fig.3a,b that higher ELM frequency and lower core radiation level are correlated. In fact, ELMs frequency linearly increases with power through separatrix above a given threshold (Fig.3d), which is a typical behavior of type-I ELMs (note that the threshold $P \approx 6 \mathrm{MW}$ is close to the $\mathrm{H}$ to $\mathrm{L}$ power threshold calculated with Martin's scaling law [32]). Thus, it could also be that ELM frequency is not directly controlled by fueling in these set of experiments, but rather set by power 


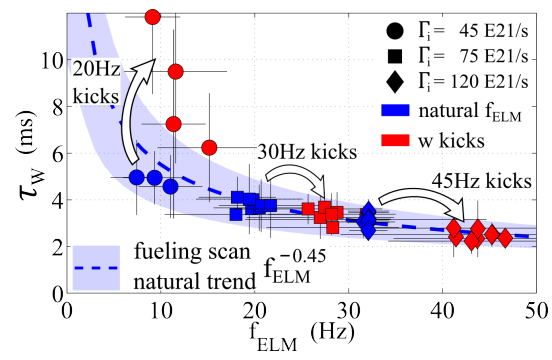

Figure 4: Variation of tungsten penetration factor versus ELM frequency for $B_{T}=2.1$ scenario during the fueling scan (blue trend). Three shot to shot comparisons of the effect of kicks are shown. Each point is a 1 s average during $\mathrm{H}$-mode plateau.

losses. Increasing fueling lowers the tungsten source, lowering the core tungsten contamination, in turns increasing the power losses. Tungsten content in the confined region increases non-linearly (almost quadratically) with the neutral W divertor source, because of the pedestal flushing decreasing with increasing core radiation. Divertor screening may also participate in the process $[13 ; 9]$, which should be focused in future analyses of these experiments.

\subsection{Vertical kicks}

To consolidate the interpretation of the correlation between penetration factor and ELM frequency, a subset of pulses from the fueling scan at $B_{T}=2.1 \mathrm{~T}$ have been identically repeated, with vertical kicks starting at H-mode formation. It should be noted that at given fueling rate, both time averaged recycling ion flux and pedestal profiles were not directly modified by the application of vertical kicks. For relatively high natural ELM frequency $\left(f_{E L M} \geq 20 \mathrm{~Hz}\right)$, vertical kicks have a good probability $\left(p_{\text {trig }} \geq 80 \%\right)$ to trigger ELMs at a frequency $50 \%$ above the natural one. As shown in Fig. $4, \tau_{W}$ obtained during two series of kicks (respectively $30 \mathrm{Hw} \& 45 \mathrm{~Hz}$ ) are statistically similar to $\tau_{W}$ obtained at same $f_{E L M}$ with recycling alone. It could confirm the control of tungsten penetration with ELM frequency, although it is observed in a domain where ELM frequency is high and tungsten radiation is already low. At lower natural ELM frequency $f_{E L M} \approx 10 \mathrm{~Hz}$ obtained at lower fueling/recycling, the application of $20 \mathrm{~Hz}$ kicks was unssuccessful to trigger ELMs $\left(p_{\text {trig }} \approx 60 \%\right)$ even at the beginning of H-mode. For currently unexplained reasons, plasma radiation reached significantly higher level than without kicks, in two consecutive pulses. It may involve low divertor screening at low fueling, or non monitored tungsten sources induced by kicks (tungsten dust). Note also that pedestal temperature droped from $\approx 700 \mathrm{eV}$ to $\approx 500 \mathrm{eV}$ after 1 s of kicks, further preventing ELM triggering at higher pedestal collisionality [24].

\subsection{Beryllium evaporation}

In order to reduce the apparent tungsten areas facing the plasma, a beryllium evaporation was applied in the main chamber. A set of pulses were repeated after the evaporation with identical control parameters (fueling, heating, etc). An illustration is shown in Fig.5a. Beryllium evaporation leads to a lower plasma density (20\%) for similar fueling, 


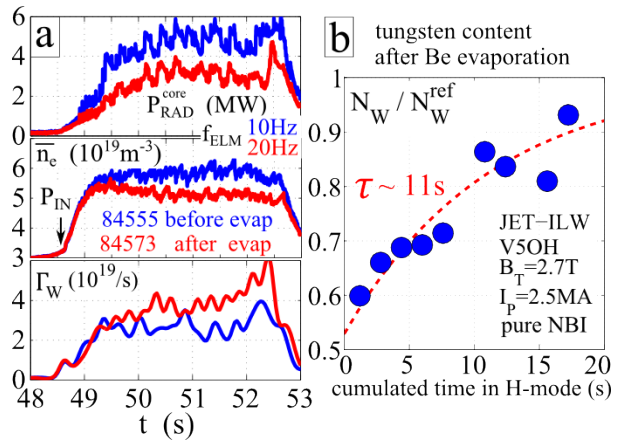

Figure 5: a) Comparison of two NBI heated pulses before (blue) after (red) beryllium evaporation showing the core radiated power, line averaged electron density, outer divertor tungsten source. b) Evolution of core tungsten content during H-mode from the first H-mode phase after beryllium evaporation, compared to reference pulses.

probably due a higher fuel trapping in beryllium layers. As shown in Fig.5b, the effective core tungsten content is about $40 \%$ lower than reference in the first $\mathrm{H}$-mode following the evaporation, but increases toward the reference value across successive H-mode phases with a time scale in the order of 10 seconds. Because the relation between source and core radiation is probably non linear, this time scale does not correspond to the wall recovery time scale. Interestingly, the source of tungsten monitored at one toroidal location in the divertor is slighly higher after evaporation. It suggests that evaporation did not efficiently cover the divertor tungsten tiles at the measurement location. Second, we observe in Fig.3e that the proportionality between core tungsten content and divertor source is statistically lower after the Beryllium evaporation than before it, at given ELM frequency. Since the link between power losses and ELM frequency is not affected by the evaporation (Fig.3d), it means that either divertor tungsten screening has been modified, or that the local source measurement is not representative of the total source. It could be the consequence of toroidal inhomogeneity of the beryllium layer in the divertor. But it could also be that main chamber surfaces (divertor excluded), which are more efficiently covered by the beryllium evaporation, dominate the source contaminating the core plasma. In AUG, these surfaces are mostly tungsten, and corresponding sources dominate the plasma pollution with respect to divertor sources [19] due to poor screening. In JET however, main chamber tungsten areas are recessed from the plasma in these particular experiments, but could anyhow participate in the global source. This point should be further investigated because it suggests, as in AUG, that divertor source may not be the relevant quantity controlling the core pollution. This conclusion needs to be addressed with specific spectroscopic measurements in the JET-ILW main chamber.

\subsection{Central ICRH heating}

Finally we investigate tungsten transport through the core. As mentioned previously (Fig.2), a large increase of core radiation can be correlated with an increase of electron density peaking [20]. In fact, the radiation peaking is increasing as well in this example. To test the effect of electron temperature profile on impurity (or radiation) peaking, 


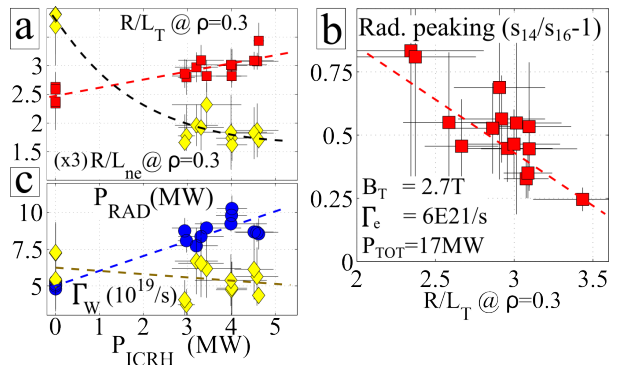

Figure 6: Influence of central ICRH heating on core plasma: a) Core electron temperature (squares) and density (diamonds) gradient vs. ICRH power. b) Central radiation peaking vs core temperature gradient. Radiation peaking is evaluated by the ratio of 2 horizontal bolometer channels (cf. fig.1). c) core radiation level (blue dots) and outer divertor tungsten source (yellow diamonds). Each point is a 2sec average during $\mathrm{H}$-mode in order to smooth saw-tooth induced modulation.

central ICRH is applied. A set of pulses is selected, with similar fueling rate $\Gamma_{e} \approx$ $5.5 E 21 \mathrm{~s}^{-1}$, similar heating power $P_{T O T} \approx 17 \mathrm{MW}$ but varying ICRH fraction ( 0 to $25 \%$ ). As shown in Fig.6a, central ICRH heating increases the electron temperature gradient by about $40 \%$ with $25 \%$ of heating provided by ICRH, whereas electron density gradient decreases by more than 50\%. Both effects are supposed to either decrease the inward pinch or increase outward impurity flow [33], which is indeed suggested by the strong decrease (by a factor of 3) of central radiation peaking shown in Fig.6b. This peaking is simply calculated from the ratio of two channels of an horizontal bolometer, one passing by the plasma center $(\rho=0)$, one passing above at $\rho=0.3$ (see Fig.1a). A concomitant effect of ICRH is a global increase of core radation (from 5 to $10 \mathrm{MW}$ with $4 \mathrm{MW}$ of ICRH), although the outer divertor tungsten source does not show systematic increase (see Fig.6c). This behavior of core radiation is investigated in [34] for other JET-ILW discharges. It is shown that ICRH increases the concentration of both tungsten and nickel (from Inconel structure material) in the confined plasma region, and that tungsten dominates the total radiation. The control of central density and temperature profiles with ICRH offers in any case a control actuator to avoid central tungsten accumulation, as demonstrated in AUG [35].

\section{Conclusion and discussion}

A series of experiments have been conducted on JET-ILW do identify the relevant physical mechanisms involved in the control of tungsten sources and transport. Tungsten accumulation at the very center of the confined plasma can be mitigated with sufficient amount of ICRH. Flattening of the central radiation profile is correlated with a peaking of the electron temperature and a flattening of the electron density profile. However the application of ICRH leads to an increase of the global radiation level. The remaining of the analyses focused on the control of edge transport and divertor sources. In non detached divertor conditions with type I ELMs, the increase of gas fueling leads to: (1) increase of ion recycling flux at divertor (2) decrease of the tungsten source at divertor (3) increase of ELM frequency (4) decrease of core radiation. These correlations make the interpretation of fueling scan experiments quite challenging. They have 
been complemented with ELM frequency control experiments, using vertical kicks, and a Beryllium evaporation to cover exposed tungsten surfaces. The decrease of the tungsten divertor source with fueling is probably due to the strong decrease of sputtering yield at lower divertor temperature. The core tungsten content decreases in correlation with the divertor source. Interestingly, the core response to the source is non-linear. The $\mathrm{W}$ penetration factor, or ratio of core tungsten content to divertor source, decreases by several units with increasing gas fueling. Both ELM frequency and divertor collisionality increase with increasing gas fueling, so that pedestal flushing and divertor screening are possibly responsible. For medium to high gas fueling levels, vertical kicks were able to increase the ELM frequency by $50 \%$. Correlated is a statistically lower core radiation level, or $\mathrm{W}$ penetration factor, following the trend built across the fueling scan alone. It may suggest that pedestal flushing is responsible for this behavior, but more analyses on divertor conditions have to be done. At lower gas fueling, kicks were neither successful in increasing ELM frequency nor reducing core radiation level. Beryllium evaporation leads to a $50 \%$ reduction of core radiation in the first $\mathrm{H}$-mode, but the improvement vanishes after approximatelvely 10 seconds of cummulated H-modes. The tungsten source measured in the divertor was however not reduced, so that the $\mathrm{W}$ penetration factor was lower than before evaporation. Possibly, the divertor is not the relevant source location dominating core pollution.

Tungsten source and transport respond significantly to modification of the plasma background, and in turns impact the plasma due to the high radiation capability of tungsten. An example is the sensitive ELM frequency response to core radiation at given input power, or the impact of power losses on the divertor temperature and so tungsten source. Coupling mechanisms between core and plasma boundary are of primary importance in the control of core tungsten radiation, and should be better understood to define optimum control schemes.

\section{Acknowledgment}

This work was supported by EURATOM and carried out within the framework of the European Fusion Development Agreement. The views and opinions expressed herein do not necessarily reflect those of the European Commission.

\section{References}

[1] R.A. Pitts et al. J. Nucl. Mater., 438(0):S48 - S56, 2013.

[2] T. Loarer et al. J. Nucl. Mater., 438(0):S108 - S113, 2013.

[3] G F Matthews and EFDA-JET contributors. Physica Scripta, 2011(T145):014001, 2011.

[4] J.W. Coenen et al. J. Nucl. Mater., 438, Supplement(0):S27 - S33, 2013.

[5] A. Kallenbach and ASDEX Upgrade Team. Nuclear Fusion, 49(4):045007, 2009.

[6] H. Zohm and et al. Nuclear Fusion, 49(10):104009, 2009.

[7] E. Joffrin and JET-EFDA Contributors. Nuclear Fusion, 54(1):013011, 2014.

[8] Vl.V. Bobkov and ASDEX Upgrade Team. Nuclear Fusion, 50(3):035004, 2010.

[9] G.J. van Rooij et al. J. Nucl. Mater., 438, Supplement(0):S42 - S47, 2013.

[10] R. Dux et al. J. Nucl. Mater., 363-365(0):112 - 116, 2007.

[11] Vl. Bobkov et al. Journal of Nuclear Materials, 415(1):S1005 - S1008, 2011.

[12] A V Chankin, D P Coster, and R Dux. Plasma Physics and Controlled Fusion, 56(2):025003, 2014.

[13] A Järvinen and JET-EFDA contributors. Physica Scripta, 2011(T145):014013, 2011.

[14] R. Dux and ASDEX Upgrade Team. Nuclear Fusion, 51(11):119501, 2011. 
[15] M. E. Puiatti and JET-EFDA contributors. Physics of Plasmas, 13(4):-, 2006.

[16] A. Jarvinen et al. J. Nucl. Mater., 438(0):S1005 - S1009, 2013.

[17] A Loarte and EFDA-JET Workprogramme Collaborators. Plasma Phys. Control. Fusion, 44(9):1815, 2002.

[18] H Urano and ASDEX Upgrade Team. Plasma Phys. Control. Fusion, 45(9):1571, 2003.

[19] R. Dux et al. J. Nucl. Mater., 390-39(0):858 - 863, 2009.

[20] T. Pütterich and the ASDEX Upgrade Team. Nuclear Fusion, 50(2):025012, 2010.

[21] M.F.F. Nave, J. Rapp, T. Bolzonella, and contributors to the JET-EFDA Workprogramme. Nuclear Fusion, 43(10):1204, 2003.

[22] T Pütterich, the ASDEX Upgrade Team, and JET EFDA Contributors. Plasma Phys. Control. Fusion, 55(12):124036, 2013.

[23] T. Nicolas et al. Physics of Plasmas, 21(1):-, 2014

[24] E. de la Luna et al. on JET Proc. 24th IAEA Fusion Energy Conf, 2012.

[25] E. Lerche et al. this conference, P1-061, 2014.

[26] K Behringer, H P Summers, B Denne, M Forrest, and M Stamp. Plasma Phys. Control. Fusion, 31(14):2059, 1989.

[27] N. Den Harder and JET-EFDA contributors. This conference, 2014.

[28] J.W. Coenen et al. This conference, 2014.

[29] T Pütterich and the ASDEX Upgrade Team. Plasma Phys. Control. Fusion, 50(8):085016, 2008.

[30] T. Pütterich and JET-EFDA Contributors. Proceedings of 24th IAEA Fusion Energy Conference (San Diego, USA), page pp. EXP3.

[31] B. Lipschultz, D.A. Pappas, B. LaBombard, J.E. Rice, D. Smith, and S.J. Wukitch. Nuclear Fusion, $41(5): 585,2001$

[32] Y R Martin, T Takizuka, and the ITPA CDBM H-mode Threshold Database Working Group. Journal of Physics: Conference Series, 123(1):012033, 2008.

[33] M. Valisa and JET-EFDA contributors. Nuclear Fusion, 51(3):033002, 2011.

[34] Czarnecka and JET-EFDA contributors. AIP Conference Proceedings, 1580(1):227-230, 2014.

[35] R. Neu and the ASDEX Upgrade team. Nuclear Fusion, 45(3):209, 2005. 